\title{
The curvature of the conical intersection seam: An approximate second-order analysis
}

\author{
Martin J. Paterson, Michael J. Bearpark, and Michael A. Robb ${ }^{\text {a) }}$ \\ Department of Chemistry, Imperial College, London SW7 2AZ, United Kingdom \\ Lluís Blancafort \\ Institut de Química Computacional and Departament de Química, Universitat de Girona, E-17071 Girona, \\ Spain
}

(Received 20 July 2004; accepted 15 September 2004)

\begin{abstract}
We present a method for analyzing the curvature (second derivatives) of the conical intersection hyperline at an optimized critical point. Our method uses the projected Hessians of the degenerate states after elimination of the two branching space coordinates, and is equivalent to a frequency calculation on a single Born-Oppenheimer potential-energy surface. Based on the projected Hessians, we develop an equation for the energy as a function of a set of curvilinear coordinates where the degeneracy is preserved to second order (i.e., the conical intersection hyperline). The curvature of the potential-energy surface in these coordinates is the curvature of the conical intersection hyperline itself, and thus determines whether one has a minimum or saddle point on the hyperline. The equation used to classify optimized conical intersection points depends in a simple way on the first- and second-order degeneracy splittings calculated at these points. As an example, for fulvene, we show that the two optimized conical intersection points of $C_{2 v}$ symmetry are saddle points on the intersection hyperline. Accordingly, there are further intersection points of lower energy, and one of $C_{2}$ symmetry - presented here for the first time-is found to be the global minimum in the intersection space. (C) 2004 American Institute of Physics.
\end{abstract}

[DOI: $10.1063 / 1.1813436]$

\section{INTRODUCTION}

Conical intersections (CIs) have been shown to play an essential role in the radiationless decay processes involved in photochemistry. ${ }^{1-8}$ At the simplest level, a conical intersection appears as a funnel in the two coordinates that lift the degeneracy. However, we know that an intersection is in fact a hyperline, i.e., a $(3 N-8)$-dimensional space where $(3 N$ -6 ) is the number of vibrational degrees of freedom, and that the "conical intersections" we optimize with gradientdriven algorithms are critical points in this $(3 N-8)$-dimensional space. In the many examples we have studied to date, we have usually been able to infer that these optimized conical intersection points are minima in the intersection space (IS), but until now we have not been able to prove this by doing a frequency calculation, in the way that one can for a single Born-Oppenheimer surface. Now that on-the-fly dynamics is possible, ${ }^{9-13}$ computations are beginning to explore the nature of the intersection hyperline away from its minimum and show that these higher-energy regions of a conical intersection hyperline can be chemically significant. Furthermore, algorithms have been developed to map out (minimum-energy path) segments of the hyperline explicitly. ${ }^{14}$ The purpose of this paper is to show that one can develop an equation for the energy as a function of a set of curvilinear coordinates where the degeneracy is preserved to second order (i.e., the conical intersection hyperline). The curvature of the potential-energy surface in these coordinates is the curvature of the conical intersection hyperline itself,

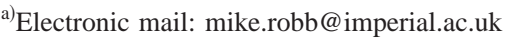

and thus determines whether one has a minimum or saddle point on the hyperline. The resulting equation used to classify optimized conical intersection points depends in a simple way on the first- and second-order degeneracy splittings calculated at these points.

In general terms, we propose a treatment of the $(3 \mathrm{~N}$ -8)-dimensional hyperline analogous to the one used for the characterization of Born-Oppenheimer surfaces, where stationary points are classified as minima or saddle points with the help of the nuclear Hessian. One immediate application is to the characterization of symmetry-restricted, optimized points of conical intersection. Thus for an optimized CI structure of a given symmetry, we are able to predict whether there are related "CI points" (i.e., critical pointsmaxima and minima-lying on the same conical intersection hyperline), which may have lower symmetry and lower energy. As a demonstration, we will characterize the optimized $S_{0} / S_{1}$ critical points on the conical intersection hyperline of fulvene. ${ }^{15}$ We will show that there are several CI critical points of different symmetry $\left(C_{2 v}, C_{s}\right.$, and $\left.C_{2}\right)$ that are minima or saddle points on the conical intersection hyperline. With the methodology that will be described in subsequent sections, we have characterized the $C_{2} \mathrm{CI}$ of fulvene as the global minimum of the intersection space for the first time, and have rationalized the interconnection of the different stationary points on the global potential-energy surface. In the future, these techniques can be combined with methods already developed ${ }^{14}$ to document minimum-energy paths (intrinsic reaction coordinates) in the intersection space.

To introduce the characterization of an optimized conical intersection point, we start from the so-called "first-order" 
approach. Thus according to the von Neumann-Wigner theorem, ${ }^{16}$ at a conical intersection, there are two degrees of freedom that lift the degeneracy at first order. This leads to the usual characterization of a conical intersection in terms of two degeneracy-lifting coordinates that form the branching space $(\mathrm{BS})$, and the remaining $(3 N-8)$-dimensional intersection space coordinates.

The degeneracy at a crossing point can also be lifted at second order. However, we can choose a coordinate system in which to mix the branching and intersection space coordinates to remove this splitting and preserve the degeneracy to second order. These new coordinates are curvilinear rather than rectilinear. We are interested in the curvature of the potential-energy surface in these coordinates, since this gives the curvature of the conical intersection hyperline and determines whether one has a minimum or saddle point on the hyperline.

As we will show, this second-order analysis can be carried out starting from the intersection space Hessians, after elimination of the branching space coordinates by projection. The gradient is zero in the intersection space at an optimized (stationary) point on a conical intersection hyperline, and the diagonalization of the Hessian yields $(3 N-8)$ vibrational frequencies. However, we have two Hessians in the intersection space and thus two sets of vibrational frequencies; one for each of the two degenerate components. In the simple "first-order" picture, we assume that the two intersecting states will have identical Hessians. However, as we will show, the Hessians of the two states are different because of second-order effects. The two surfaces split as one moves away from the optimized CI point along intersection space coordinates (second order) as well as along the branching space coordinates (first order). As we shall discuss, this situation is analogous to the well-known Renner-Teller picture for a linear molecule. In order to preserve the degeneracy of the conical intersection correct to second order, the conical intersection hyperline must bend as the branching and intersection space coordinates mix along a curvilinear coordinate. Thus, at second order, the analysis of the Hessian in the intersection space demonstrates how these effects change the usual first-order picture.

We proceed now to a mathematical development before illustrating the central concepts and demonstrating that the method can yield new results using fulvene as an example. The next two parts of the paper have been written so that they can be read in any order.

\section{QUADRATIC REPRESENTATION OF THE POTENTIAL-ENERGY SURFACE IN THE REGION OF A CONICAL INTERSECTION}

From a practical point of view, we start with the Hessians of the two degenerate states at an optimized conical intersection point. We use an initial set of branching and intersection space coordinates that are assumed to have come from the diagonalization of these Hessians. Our development will be based on a (simplified) Taylor expansion (to second order) taken over from the spectroscopically oriented treatment of conical intersections. ${ }^{17-19}$ The conical intersection line itself, correct to second order, becomes a paraboloid or a hyperboloid. Consequently, the characterization of the conical intersection line correct to second order requires curvilinear coordinates, which are nonlinear combinations of the branching and intersection space coordinates. The curvature of the conical intersection hyperline is therefore determined by the second derivatives with respect to these curvilinear coordinates.

The degeneracy is lifted in first-order nuclear displacements via the branching space coordinates; the gradient difference (GD) vector [Eq. (1a)] and the interstate coupling vector [Eq. (1b)],

$$
\begin{aligned}
& x_{1}=\frac{\partial\left(E_{B}-E_{A}\right)}{\partial \xi}, \\
& x_{2}=\left\langle\Psi_{A}\left|\frac{\partial \hat{H}_{e}}{\partial \xi}\right| \Psi_{B}\right\rangle,
\end{aligned}
$$

where $\xi$ is a vector of Cartesian displacements, $\Psi_{A}$ and $\Psi_{B}$ are the adiabatic electronic wave functions, and $\hat{H}_{e}$ is the clamped nucleus electronic Hamiltonian operator. (These two vectors are used in algorithms for locating optimized points on the conical intersection hyperline. ${ }^{20,21}$ ) The branching space is sometimes referred to as the $\mathrm{g}$-h plane. ${ }^{3-5}$

We now introduce a set of coordinates to represent the potential-energy surface in the region of a critical point on a conical intersection seam,

$$
\bar{Q}=\left(\bar{Q}_{x_{1}}, \bar{Q}_{x_{2}}\right) \oplus\left(\bar{Q}_{1}, \ldots, \bar{Q}_{3 N-8}\right)
$$

The branching space is spanned by the mass-weighted gradient difference vector $\left(\bar{Q}_{x_{1}}\right)$, and by the mass-weighted interstate coupling vector $\left(\bar{Q}_{x_{2}}\right)$. The orthogonal complement space (the intersection space ${ }^{1}$ ) is spanned by $\left(\bar{Q}_{1}, \ldots, \bar{Q}_{3 N-8}\right)$. The potential-energy surface for ground and excited states is obtained by diagonalizing $V$,

$$
\begin{aligned}
& V=\left(\begin{array}{cc}
E^{A} & V_{12} \\
V_{21} & E^{B}
\end{array}\right)=V^{1}+V^{2}=V^{1}+V_{a}^{2}+V_{b}^{2}+V_{c}^{2}, \\
& V^{1}=\left(\begin{array}{cc}
\kappa^{A} \bar{Q}_{x_{1}} & \kappa^{A B} \bar{Q}_{x_{2}} \\
\kappa^{A B} \bar{Q}_{x_{2}} & \kappa^{B} \bar{Q}_{x_{1}}
\end{array}\right), \\
& V_{a}^{2}=\left(\begin{array}{c}
\sum_{i, j \in B S} \gamma_{i j}^{A} \bar{Q}_{i} \bar{Q}_{j} \quad \sum_{i, j \in B S} \eta_{i j}^{A B} \bar{Q}_{i} \bar{Q}_{j} \\
\sum_{i, j \in B S} \eta_{i j}^{A B} \bar{Q}_{i} \bar{Q}_{j} \sum_{i, j \in B S} \gamma_{i j}^{B} \bar{Q}_{i} \bar{Q}_{j}
\end{array}\right),
\end{aligned}
$$




$$
\begin{aligned}
& V_{b}^{2}=\left(\begin{array}{cc}
\sum_{i \in B S, j \in I S} \gamma_{i j}^{A} \bar{Q}_{i} \bar{Q}_{j} & \sum_{i \in B S, j \in I S} \eta_{i j}^{A B} \bar{Q}_{i} \bar{Q}_{j} \\
\sum_{i \in B S, j \in I S} \eta_{i j}^{A B} \bar{Q}_{i} \bar{Q}_{j} & \sum_{i \in B S, j \in I S} \gamma_{i j}^{B} \bar{Q}_{i} \bar{Q}_{j}
\end{array}\right), \\
& V_{c}^{2}=\left(\begin{array}{cc}
\sum_{i, j \in I S} \gamma_{i j}^{A} \bar{Q}_{i} \bar{Q}_{j} & \sum_{i, j \in I S} \eta_{i j}^{A B} \bar{Q}_{i} \bar{Q}_{j} \\
\sum_{i, j \in I S} \eta_{i j}^{A B} \bar{Q}_{i} \bar{Q}_{j} & \sum_{i, j \in I S} \gamma_{i j}^{B} \bar{Q}_{i} \bar{Q}_{j}
\end{array}\right) .
\end{aligned}
$$

The potential constants above, $\kappa^{A}, \kappa^{B}, \kappa^{A B}, \gamma_{i j}^{A}, \gamma_{i j}^{B}$, and $\eta_{i j}^{A B}$, are defined by the following equations:

$$
\begin{aligned}
& \kappa^{I}=\left\langle\Psi_{I}\left|\left(\frac{\partial \hat{H}_{e}}{\partial \bar{Q}_{x_{1}}}\right)_{0}\right| \Psi_{I}\right\rangle, \\
& \kappa^{A B}=\left\langle\Psi_{A}\left|\left(\frac{\partial \hat{H}_{e}}{\partial \bar{Q}_{x_{2}}}\right)_{0}\right| \Psi_{B}\right\rangle, \\
& \gamma_{i j}^{I}=\left\langle\Psi_{I}\left|\left(\frac{\partial^{2} \hat{H}_{e}}{\partial \bar{Q}_{i} \partial \bar{Q}_{j}}\right)_{0}\right| \Psi_{I}\right\rangle, \\
& \eta_{i j}^{A B}=\left\langle\Psi_{A}\left|\left(\frac{\partial^{2} \hat{H}_{e}}{\partial \bar{Q}_{i} \partial \bar{Q}_{j}}\right)_{0}\right| \Psi_{B}\right\rangle,
\end{aligned}
$$

where $\Psi_{A}$ and $\Psi_{B}$ are the degenerate adiabatic wave functions computed at the conical intersection point. Since we use state-averaged wave functions, these two states are rigorously orthogonal (see the discussion in Ref. 23 about the choice of degenerate wave functions and the choice of the two vectors for the branching space). Diagonalization of the potential matrix at any finite displacement along the coordinates $\bar{Q}$ gives the energies of state $A$ or $B$. The reference energy is $E_{A}^{0}$, or its equivalent $E_{B}^{0}$, the adiabatic energy at $\bar{Q}=0$ (an optimized CI point). The terms $\kappa^{A}, \kappa^{B}$, and $\kappa^{A B}$ are just the gradients and the interstate coupling, which are computed during a conical intersection optimization. The second-order interstate coupling terms $\eta_{i j}^{A B}$ could be computed. However, in this paper we base our analysis on the diagonal terms $\gamma_{i i}^{A}$ and $\gamma_{i i}^{B}$, which can be obtained from a frequency calculation in the reduced $(3 N-8)$-dimensional intersection space.

We now discuss the interpretation of the first- and second-order terms $\kappa$ and $\gamma_{i j}^{I}, \eta_{i j}^{A B}$, respectively, with the help of the partition of $V$ [Eq. (3)]. The first-order part of $V$, $V^{1}$, contains the first-order terms $\kappa$. It is clear that for any displacement in the branching space $\left(\bar{Q}_{x_{1}}, \bar{Q}_{x_{2}}\right)$, the degeneracy is lifted (to first order) via $V^{1}$. Notice that we assume that we are expanding about an optimized point on the conical intersection, so the gradient terms occur only in the branching space (gradient difference along $\bar{Q}_{x_{1}}$ and interstate coupling along $\bar{Q}_{x_{2}}$ ). For a displacement in the intersection space $\left(\bar{Q}_{1}, \ldots, \bar{Q}_{3 N-8}\right)$, the degeneracy remains to first order

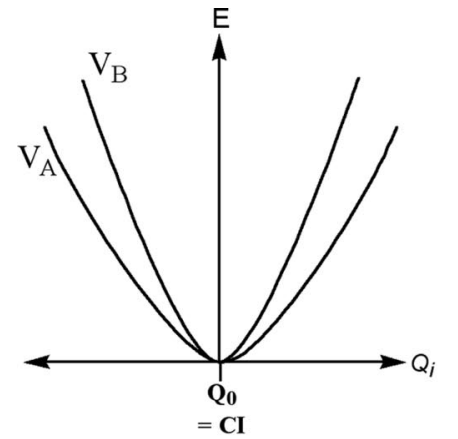

Renner-Teller

Type I

${ }^{c} \gamma_{i}^{A}>0,{ }^{c} \gamma_{i}^{B}>0$

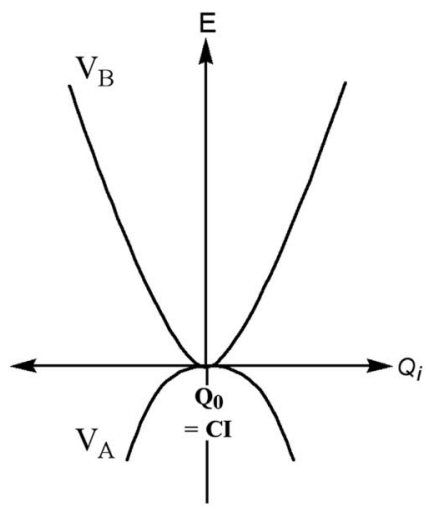

Renner-Teller

Type II

${ }^{c} \gamma_{i}^{A}<0,{ }^{c} \gamma_{i}^{B}>0$

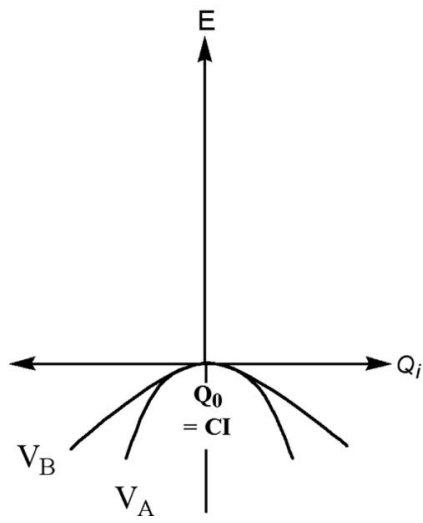

Renner-Teller

Type III

${ }^{c} \gamma_{i}^{A}<0,{ }^{c} \gamma_{i}^{B}<0$

FIG. 1. Classification of Renner-Teller-type profiles along the intersection space rectilinear coordinates.

but is, in general, lifted at second order through the terms in $V^{2}$ if all the $\gamma_{i j}^{A}$ are not equal to the $\gamma_{i j}^{B}$ and/or the $\eta_{i j}^{A B}$ are not zero. The second-order terms are partitioned in three groups: $V_{a}^{2}$ includes effects along the branching space modes, $V_{b}^{2}$ includes effects between the branching and intersection space modes, and $V_{c}^{2}$ includes effects along the intersection space modes. For simplicity, we shall use ${ }^{a} \lambda_{i j}^{A}$ for terms $\lambda_{i j}^{A}$ $\in V_{a}^{2}$, etc.

To keep the development simple, we shall now introduce some approximations. We discuss the validity of some of these approximations subsequently. First, we assume that all so-called cross-quadratic terms $\gamma_{i j}(i \neq j)$ and all secondorder interstate couplings $\eta_{i j}^{A B}$ are zero. Thus, $V_{b}^{2}$ can be neglected, and the remaining second-order parts are simplified. Of course, in general, $\gamma_{i j}^{A} \neq \gamma_{i j}^{B}$ so that one has different sets of eigenvectors for the two states. In practice, the eigenvectors for two states are almost identical, and it is the eigenvalues or diagonal elements that are different. The as- 
sumption that all $\eta_{i i}^{A B}$ terms are zero is rigorous for the fulvene example discussed below, because the so-called second-order interstate couplings are zero by symmetry. Thus, we are left with a simplified quadratic form that contains only diagonal second-order terms,

$$
\begin{aligned}
V= & E+\left(\begin{array}{cc}
\kappa^{A} \bar{Q}_{x_{1}} & \kappa^{A B} \bar{Q}_{x_{2}} \\
\kappa^{A B} \bar{Q}_{x_{2}} & \kappa^{B} \bar{Q}_{x_{1}}
\end{array}\right) \\
& +\left(\begin{array}{cc}
\sum_{i \in B S}{ }^{a} \gamma_{i i}^{A} \bar{Q}_{i}^{2} & 0 \\
0 & \sum_{i \in B S}{ }^{a} \gamma_{i i}^{B} \bar{Q}_{i}^{2}
\end{array}\right) \\
& +\left(\begin{array}{cc}
\sum_{i \in I S}{ }^{c} \gamma_{i i}^{A} \bar{Q}_{i}^{2} & 0 \\
0 & \sum_{i \in I S}{ }^{c} \gamma_{i i}^{B} \bar{Q}_{i}^{2}
\end{array}\right),
\end{aligned}
$$

where $E$ is a diagonal matrix with diagonal elements equal to $E_{A}^{0}$ and $E_{B}^{0}$ (energies at the conical intersection point). This form is useful because the gradient terms are zero in the intersection space. One can obtain new insights by carrying out a standard frequency analysis and by calculating the force constants ${ }^{c} \gamma_{i i}^{A}$ and ${ }^{c} \gamma_{i i}^{B}$ in this space.

Let us digress at this stage and discuss the interpretation that one might make of a frequency analysis for each state within the intersection space. From a conceptual point of view, it is possible to distinguish three different cases for the frequencies that might be obtained. In the intersection space, the conical intersection behaves like a Renner-Teller intersection of a linear molecule in an orbitally degenerate state; the gradient of each state is zero, and the degeneracy is lifted quadratically. The various possibilities are shown in Fig. $1{ }^{22}$

However, interpretation of the frequency analysis within an intersection space on the basis of Fig. 1 is by no means straightforward. If the curvature of both surfaces is the same, then one is tempted to imagine that the optimized point on the conical intersection hyperline is a maximum or minimum. However, when the curvature of both surfaces in the intersection space is different, there is no obvious way to

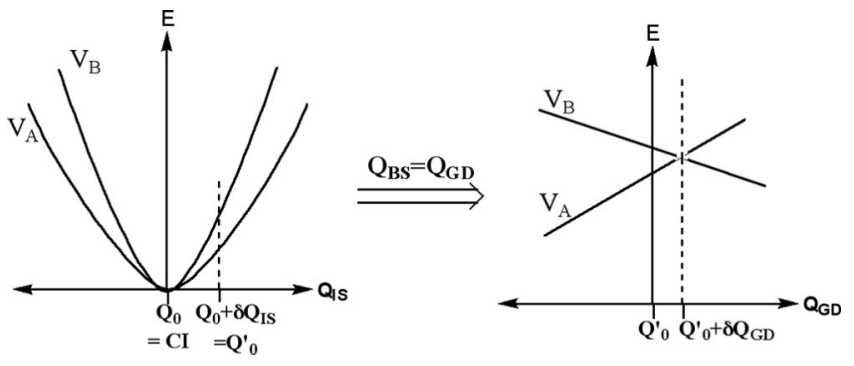

FIG. 2. Effect of consecutive displacements from the conical intersection along one IS mode and the GD mode of the BS.

guess the curvature of the conical intersection hyperline itself. The conceptual problem is resolved only when one moves from rectilinear coordinates to curvilinear coordinates.

Before these curvilinear coordinates are expressed mathematically, we develop this idea intuitively as a combination of first- and second-order degeneracy-lifting effects. As we have just discussed in Fig. 1, an infinitesimal displacement $\delta \bar{Q}_{I S}$ along one of the intersection space coordinates produces a splitting of the surfaces equivalent to the difference between the two eigenvalues (see also the left-hand side of Fig. 2, where the effect is shown using finite displacements). However, a subsequent infinitesimal displacement $\delta \bar{Q}_{B S}$ along a branching space coordinate, namely, the gradient difference, can eventually recover the degeneracy by bringing the energies of the two states together again. Thus, the new degeneracy-retaining coordinate is realized as a combination of the two displacements, and a new set of degeneracyretaining coordinates can be defined as combinations of one intersection space and one branching space coordinate (the gradient difference).

\section{CHARACTERIZATION OF THE SEAM: DEFINITION OF A CURVILINEAR COORDINATE SYSTEM AND CALCULATION OF THE SEAM CURVATURE}

Our purpose in this section is to develop the working equations for the characterization of the conical intersection using the simplified quadratic form developed previously [Eq. (8)]. After diagonalization of $V$, the energies of the two states can be expressed as

$$
\begin{aligned}
& E_{A, B}=\lambda \bar{Q}_{x_{1}}+\sum_{i \in B S}{ }^{a} \omega_{i} \bar{Q}_{i}^{2}+\sum_{i \in I S}{ }^{c} \omega_{i} \bar{Q}_{i}^{2} \pm \frac{1}{2} \sqrt{\left(\delta \kappa \bar{Q}_{1}+\sum_{i \in B S}{ }^{a} \delta \gamma_{i} \bar{Q}_{i}^{2}+\sum_{i \in I S}{ }^{c} \delta \gamma_{i} \bar{Q}_{i}^{2}\right)^{2}+\left(2 \kappa^{A B} \bar{Q}_{x_{2}}\right)^{2}}, \\
& \lambda=\left(\kappa^{B}+\kappa^{A}\right) / 2, \\
& \delta \kappa=\kappa^{B}-\kappa^{A}, \\
& \omega_{i}=\left(\gamma_{i i}^{B}+\gamma_{i i}^{A}\right) / 2, \\
& \delta \gamma_{i}=\gamma_{i i}^{B}-\gamma_{i i}^{A} .
\end{aligned}
$$

Thus the energy difference between the two states is 


$$
\Delta E=\sqrt{\left(\delta \kappa \bar{Q}_{x_{1}}+\sum_{i \in B S}{ }^{a} \delta \gamma_{i} \bar{Q}_{x_{i}}^{2}+\sum_{i \in I S}{ }^{c} \delta \gamma_{i} \bar{Q}_{i}^{2}\right)^{2}+\left(2 \kappa^{A B} \bar{Q}_{\mathbf{x}_{2}}\right)^{2}} .
$$

In this expression, it is clear that the energy splitting between the intersecting states comes from first-order effects along the branching space coordinates $x_{1}$ and $x_{2}$ and from quadratic second-order effects along all coordinates. Moreover, by assuming that all cross-quadratic terms $\gamma_{i j}(i \neq j)$ between branching and intersection space coordinates are zero and that second-order interstate couplings $\eta_{i j}$ are zero, we have the tacit assumption that all energy splittings that appear as differences in the eigenvalues of the two intersection space Hessians come from differences in the force constants of the two states within the intersection space itself.

We now proceed to derive the equation for the conical intersection hyperline correct to second order. We begin by setting the energy difference in Eq. (10) to zero. This gives the condition for the curvilinear coordinates that retain the energy degeneracy. These coordinates are used to obtain an expression for the energy of the seam as a function of the curvilinear coordinates $\left\{t_{i}\right\}$. This expression is finally used to characterize the seam by its second derivatives, $\left(\partial^{2} E / \partial t_{i}^{2}\right)_{t_{i}=0}$.

For our remaining analysis, we introduce one more simplification, namely, we neglect the quadratic splittings along the branching space modes, ${ }^{a} \delta \gamma_{i}$. In fact, the inclusion of these terms would complicate the following development but does not change the conclusions. As we will show, the ${ }^{a} \delta \gamma_{i}$ terms affect the magnitude of $\left(\partial^{2} E / \partial t_{i}^{2}\right)_{t_{i}=0}$ but not its sign, which is our main point of interest (see Appendix for the details of including $\left.{ }^{a} \delta \gamma_{i}\right)$. Neglecting the ${ }^{a} \delta \gamma_{i}$ splitting and setting the energy difference [Eq. (10)] to zero, one obtains Eq. (11),

$$
\left(\delta \kappa \bar{Q}_{x_{1}}+\sum_{I S}{ }^{c} \delta \gamma_{i} \bar{Q}_{i}^{2}\right)^{2}+\left(2 \kappa^{A B} \bar{Q}_{x_{2}}\right)^{2}=0 .
$$

From Eq. (11), the curvilinear coordinates $\left\{t_{i}\right\}$ will be combinations of the intersection space coordinates with the gradient difference vector. (If the second-order interstate coupling is included, then the interstate coupling coordinate of the branching space mixes as well.) Thus Eq. (11) is simplified to

$$
\delta \kappa \bar{Q}_{x_{1}}+\sum_{I S}{ }^{c} \delta \gamma_{i} \bar{Q}_{i}^{2}=0 .
$$

Thus, the equation of the seam is a paraboloid. There are $(3 N-8)$ solutions to this equation, which are linear combinations of the $(3 N-8)$ linearly independent intersection space modes with the $\bar{Q}_{x_{1}}$ coordinate (gradient difference). Each curvilinear coordinate $t_{i}$ is obtained as a solution to Eq. (13),

$$
\delta \kappa \bar{Q}_{x_{1}}+{ }^{c} \delta \gamma_{i} \bar{Q}_{i}^{2}=0 .
$$

We proceed by writing Eq. (13) as a function of the parameter $t$.

$$
\begin{aligned}
& \bar{Q}_{x_{1}}=-\frac{1}{\delta \kappa} t_{i}^{2}=-\alpha t_{i}^{2}, \quad \alpha=\frac{1}{\delta \kappa}, \\
& \bar{Q}_{i}=\frac{1}{\sqrt{{ }^{c} \delta \gamma_{i}}} t_{i}=\beta_{i} t_{i}, \quad \beta_{i}=\frac{1}{\sqrt{{ }^{c} \delta \gamma_{i}}} .
\end{aligned}
$$

The expression for the energy of one of the states along the $\bar{Q}_{x_{1}}$ and $\bar{Q}_{i}$ coordinates is obtained from Eq. (9) and is

$$
\begin{aligned}
E_{A} & =\lambda \bar{Q}_{x_{1}}+{ }^{a} \gamma_{1} \bar{Q}_{x_{1}}^{2}+{ }^{c} \gamma_{i} \bar{Q}_{i}^{2}+\delta \kappa \bar{Q}_{x_{1}}+{ }^{c} \delta \gamma_{i} \bar{Q}_{i}^{2} \\
& =\kappa^{A} \bar{Q}_{x_{1}}+{ }^{a} \gamma_{11}^{A} \bar{Q}_{x_{1}}^{2}+{ }^{c} \gamma_{i i}^{A} \bar{Q}_{i}^{2} .
\end{aligned}
$$

Substituting from Eqs. (15) and (16) we have

$$
\begin{aligned}
E_{A} & =-\alpha \kappa^{A} t_{i}^{2}+{ }^{a} \gamma_{11}^{A} \alpha^{2} t_{i}^{4}+{ }^{c} \gamma_{i i}^{A} \beta_{i}^{2} t_{i}^{2} \\
& ={ }^{a} \gamma_{11}^{A} \alpha^{2} t_{i}^{4}+\left({ }^{c} \gamma_{i i}^{A} \beta_{i}^{2}-\alpha \kappa^{A}\right) t_{i}^{2} .
\end{aligned}
$$

Equation (17) gives the energy of the states along a degeneracy-retaining coordinate $t_{i}$. We refer to it as the energy of the intersection seam (hyperline) along the curved coordinate $t_{i}$. The expression required to characterize the hyperline is then

$$
\left(\frac{\partial^{2} E}{\partial t_{i}^{2}}\right)_{t_{i}=0}=2\left(\gamma_{i}^{A} \beta_{i}^{2}-\kappa^{A} \alpha\right)=2\left\{\left(\frac{\gamma_{i}^{A}}{\delta \gamma_{i}}\right)-\left(\frac{\kappa^{A}}{\delta \kappa}\right)\right\} .
$$

The superscript $c$ from the $\gamma$ terms has been omitted for clarity, but it should be clear that the terms refer to the branching space coordinate (the gradient difference in the fulvene example) and the $\gamma$ terms refer to the intersection space coordinates. This gives us a working equation for the analysis of the curvature of the intersection hyperline that arises from second-order effects in the intersection space and from first-order effects along the branching space coordinates.

At this stage, we need to consider the effect of a transformation of the two degenerate wave functions at the conical intersection (see Ref. 23 for a very general discussion of this problem). If two degenerate wave functions transform as different irreducible representations of some group, then one can always make a unique choice for the two vectors of the branching space. However, when a molecule has no symmetry, the degenerate wave functions at the conical intersection are only unique to within a unitary transformation amongst themselves. Clearly the transformation of the degenerate wave functions changes or rotates the basis vectors (gradient difference and interstate coupling vectors) of the branching space. At the same time, the gradient difference and interstate coupling vectors are interconverted. Our analysis remains valid, although the $\delta \gamma_{i}$ term of Eq. (18) would be, strictly speaking, $2 \eta_{i}^{A B}$, and $\kappa^{A}$ would be the projection of the gradient of state $A$ along the new interstate coupling coordinate. 
TABLE I. Fulvene $S_{0} / S_{1}$ energetics: CASSCF $(6,6) / c c-p v D Z$.

\begin{tabular}{cccc}
\hline \hline & & & $\begin{array}{c}\text { Relative } \\
\text { energy } \\
\text { (to } \\
\text { Geometry (Table III) }\end{array}$ \\
\hline$S_{0}$ minimum & Adiabatic state & Energy/a.u. & $\begin{array}{c}S_{0} \mathrm{kcal} \mathrm{mol}^{-1} \text { ) } \\
S_{1} \text { planar minimum }\end{array}$ \\
$\mathrm{CI}_{\text {plan }}$ & $S_{0}$ & -230.7464 & 0.0 \\
$\mathrm{CI}_{\text {perp }}$ & $S_{1}$ & -230.6489 & 61.2 \\
$\mathrm{CI}_{63}$ & $S_{0} / S_{1}$ & -230.6359 & 69.4 \\
$\mathrm{CI}_{\text {pyr }}$ & $S_{0} / S_{1}$ & -230.6478 & 61.8 \\
\hline \hline & $S_{0} / S_{1}$ & -230.6514 & 59.6 \\
\hline
\end{tabular}

Thus, when there is no symmetry, and the gradient difference coordinate that occurs in Eqs. (11) and (12) is not uniquely defined, does the analysis that we have just presented remain valid? Clearly in Eqs. (11) and (12) all the quantities are differences between the values for different states. One might expect $\delta \gamma_{i}=\gamma_{i i}^{B}-\gamma_{i i}^{A}$ to be invariant to a transformation between states $A$ and $B$; we have carried out numerical tests and this seems to be the case, but as yet we have no rigorous proof.

\section{COMPUTATIONAL DETAILS}

Calculations were done at the complete active space self consistent field [CASSCF $(6,6) / c c-p V D Z]$ level of theory with a development version of GAUSSIAN99. ${ }^{24}$ The stateaveraged Hessian was computed for both roots of the degenerate state-averaged CASSCF wave function. The branching space was mass weighted and projected from each Hessian to yield two $(3 N-8)$-dimensional Hessians.

For every critical point, the normal coordinates of the two degenerate states were matched with each other by projecting one set upon the other. For the two critical $C_{2 v}$ intersection points of fulvene the normal coordinates of each surface were parallel to each other to within $1^{\circ}$ or $2^{\circ}$. This is equivalent to an accuracy of around two decimal places in the Cartesian displacement vectors. We are therefore confident that the set of coordinates used was sufficiently accurate to map out the seam of intersection qualitatively.

The valence-bond resonance structures for each component of the degenerate electronic state at all of the optimized intersections were obtained from the spin-exchange density using localized orbitals. ${ }^{25}$ See Ref. 26 for details.

\section{APPLICATION: ANALYZING THE $S_{0} / S_{1}$ SEAM IN FULVENE}

The photophysics of fulvene is characterized by a lack of fluorescence, which indicates fast internal conversion of the excited state to the ground state via a conical intersection. ${ }^{27}$ In a previous CASSCF study, two distinct critical points on the $S_{0} / S_{1}$ conical intersection seam were located: ${ }^{15}$ in $\mathbf{C I}_{\text {plan }}$, the methylene group lies in the plane of the ring, and in the other structure, $\mathbf{C I}_{\text {perp }}$, the methylene group is perpendicular to the plane. Both structures have $C_{2 v}$ symmetry, but $\mathbf{C I}_{\text {perp }}$ lies approximately $8 \mathrm{kcal} \mathrm{mol}^{-1}$ below $\mathbf{C I}_{\text {plan }}$ (Table I). The original CASSCF study of the potential-energy sur-

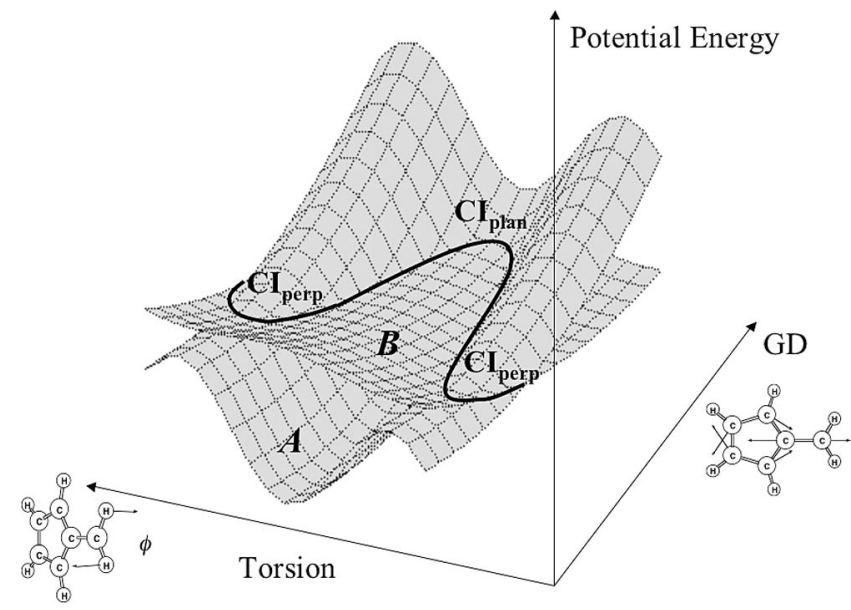

FIG. 3. Plot of the $S_{0}$ and $S_{1}$ surfaces of fulvene along the torsion and bond-inversion coordinates. The seam of intersection is marked as a bold line.

faces was complemented by a molecular mechanics valencebond (MMVB) dynamics study where the point of decay to the ground state was analyzed for many trajectories. The trajectories were found to decay at all methylene torsion angles, suggesting that the two conical intersection critical points of $C_{2 v}$ symmetry are interconnected by a continuous seam of intersection along the methylene torsion coordinate. ${ }^{15}$ (This was the first such example we studied.) We are now finally in a position to determine the curvilinear hyperline coordinate that connects the planar and twisted intersection points. Our results show that this curvilinear coordinate is composed of the torsion and bond-inversion stretching (gradient difference) coordinates, shown in Fig. 3 in bold. Torsion alone does not preserve the degeneracy, and the variation in mixing with the gradient difference along the seam leads to the curvature of the seam shown in Fig. 3.

We have characterized the two CI critical points of $C_{2 v}$ symmetry located previously as saddle points in the intersection space using Eq. (18). Table II shows (as we explain fully below) that the curvature of the CI hyperline at $\mathbf{C I}_{\text {plan }}$ is negative either when the torsion mode and the gradient difference are combined to produce the curvilinear coordinate or when the pyramidalization mode and the gradient difference mode are combined. Thus, there should exist lowerenergy CI critical points on the hyperline along these curved coordinates, and indeed, we find that the "global minimum" of the intersection space is a structure of $C_{2}$ symmetry $\left(\mathbf{C I}_{63}\right)$ with a torsion angle of $63^{\circ}$ that we had never located —or thought to look for-in our previous work.

The relative energies of the critical points (minima of the $S_{0}$ and $S_{1}$ states and optimized conical intersection points) are summarized in Table I, and the relevant geometric parameters are given in Table III. The relevant frequencies obtained from the intersection space Hessian calculation for the conical intersections of $C_{2 v}$ symmetry are listed in Table IV. (The full list of frequencies appears in EPAPS supporting information. ${ }^{28}$ )

The vibrational frequencies of the intersection space modes (i.e., the rectilinear coordinates tangent to the curvilinear seam at the optimized CI) can be understood in terms of valence-bond representations of the components of the 
TABLE II. Computed first-order parameters (projection of gradients onto gradient difference) and second-order parameters used to characterize the hyperline curvature at the four optimized fulvene CI critical points (in arbitrary units).

\begin{tabular}{|c|c|c|c|c|c|c|c|c|c|}
\hline CI & $\begin{array}{l}\text { Intersection } \\
\text { space mode }\end{array}$ & $\kappa^{A}$ & $\kappa^{B}$ & $\delta \kappa=\kappa^{B}-\kappa^{A}$ & $\begin{array}{c}\gamma_{i i}^{A} \\
\left(\times 10^{5}\right)\end{array}$ & $\begin{array}{r}\gamma_{i i}^{B} \\
\left(\times 10^{5}\right)\end{array}$ & $\begin{array}{c}\delta \gamma_{i}=\gamma_{i i}^{B}-\gamma_{i i}^{A} \\
\left(\times 10^{5}\right)\end{array}$ & $\left(\frac{\partial^{2} E}{\partial t_{i}^{2}}\right)_{t_{i}=0}=2\left\{\left(\frac{\gamma_{i}^{A}}{\delta \gamma_{i}}\right)\right.$ & $\left.\left(\frac{\kappa^{A}}{\delta \kappa}\right)\right)$ \\
\hline \multirow[t]{2}{*}{$\mathrm{CI}_{\text {Plan }}$} & Torsion & -0.03329 & -0.10164 & -0.06835 & 1.85761 & -0.32761 & -2.18522 & -2.674 & \\
\hline & Pyramidalization & & & & 1.64025 & -1.02400 & -2.66425 & -2.205 & \\
\hline \multirow[t]{2}{*}{$\mathrm{CI}_{\text {Perp }}$} & Torsion & 0.02904 & -0.02348 & -0.05251 & -10.12036 & 1.41376 & 11.53412 & -0.649 & \\
\hline & Pyramidalization & & & & -0.00400 & 0.30976 & 0.31376 & 1.080 & \\
\hline \multirow[t]{2}{*}{$\mathrm{CI}_{63}$} & Torsion & 0.05361 & -0.00652 & -0.06013 & 0.50625 & 2.53009 & 2.02384 & 2.283 & \\
\hline & Pyramidalization & & & & 2.46016 & -0.30276 & -2.76292 & 0.002 & \\
\hline \multirow[t]{2}{*}{$\mathrm{CI}_{\text {Pyr }}$} & Torsion & 0.09727 & 0.02809 & -0.06918 & -0.22500 & 1.98025 & 2.20525 & 2.608 & \\
\hline & Pyramidalization & & & & 0.96100 & 1.67281 & 0.71181 & 5.512 & \\
\hline
\end{tabular}

degenerate electronic state. These modes have Renner-Teller topologies (Fig. 1) and it is these second-order splittings that result in the seam curvature. We now discuss these secondorder splittings in detail for the two $C_{2 v}$ optimized CI critical points.

We start our analysis with the planar structures of $C_{2 v}$ symmetry (Fig. 4). At the Franck-Condon geometry, the ground state has $A_{1}$ symmetry, while the lowest singlet excited state $\left(S_{1}\right)$ has $B_{2}$ symmetry. The valence-bond structures for the two states are shown in Fig. 4. The ground state has a closed-shell structure with three localized double bonds, whereas the excited state has a diradical structure. The planar conical intersection of $C_{2 v}$ symmetry has a sloped ${ }^{1}$ topology along the gradient difference, which corresponds to symmetric bond-length inversion (recoupling or exchange of the single and double bonds). The interstate coupling is an antisymmetric stretch of the $\mathrm{C}-\mathrm{C}$ bonds.

From our intersection space Hessian calculation, we obtain the "frequencies" along the rectilinear intersection space coordinates. The second-order splittings are less than $300 \mathrm{~cm}^{-1}$ (RT-I profiles, see Fig. 1) for all modes except two. These modes are documented in Table IV and correspond to the methylene pyramidalization of $b_{1}$ symmetry and the methylene torsion of $a_{2}$ symmetry. These two modes have real frequencies for the $A_{1}$ state (i.e., positive curvature of the surface along those modes), but have imaginary values

TABLE III. Fulvene $S_{0} / S_{1}$ optimized conical intersection geometries. All bond lengths are in angstroms.

\begin{tabular}{|c|c|c|c|c|}
\hline $\begin{array}{ll}3 & 4 \\
\end{array}$ & $\begin{array}{c}\mathrm{CI}_{\text {plan }} \\
C_{2 v}\end{array}$ & $\begin{array}{c}\mathrm{CI}_{\text {perp }} \\
C_{2 v}\end{array}$ & $\begin{array}{c}\mathrm{CI}_{63} \\
C_{2}\end{array}$ & $\begin{array}{c}\mathrm{CI}_{\mathrm{pyr}} \\
C_{s}\end{array}$ \\
\hline $1-2$ & 1.372 & 1.424 & 1.409 & 1.377 \\
\hline $2-3$ & 1.531 & 1.424 & 1.461 & 1.521 \\
\hline $3-4$ & 1.320 & 1.413 & 1.371 & 1.326 \\
\hline $4-5$ & 1.531 & 1.424 & 1.461 & 1.521 \\
\hline $1-5$ & 1.372 & 1.424 & 1.409 & 1.377 \\
\hline $1-6$ & 1.578 & 1.478 & 1.481 & 1.567 \\
\hline $\begin{array}{c}\mathrm{H}_{7}-6-1-2 \\
\text { dihedral }\end{array}$ & $0.0^{\circ}$ & $90.0^{\circ}$ & $63.1^{\circ}$ & 18.1 \\
\hline
\end{tabular}

for the $B_{2}$ state (negative curvature), corresponding to RT-IItype profiles (see Fig. 1).

One can rationalize the different signs of the curvature for the two states using the valence-bond structures shown in Fig. 4. In the $A_{1}$ state, there is a $\pi$ bond between $\mathrm{C}_{1}$ and $\mathrm{C}_{6}$ and the pyramidalization and torsion modes have real frequencies. In contrast to this, in the $B_{2}$ state, the methylene group carries an uncoupled electron (i.e., a radical) and the energy is lowered by the same modes (i.e., imaginary frequencies).

Due to the different signs of the curvatures, there is a substantial second-order splitting along these modes. The degeneracy-retaining, curvilinear coordinates $\left\{t_{i}\right\}$ are combinations of these modes with the gradient difference coordinate. Substituting the computed gradients and curvatures at the intersection in Eq. (18), we calculate the value of $\left(\partial^{2} E_{s}\left(t_{i}\right) / \partial t_{i}^{2}\right)_{t_{i}=0}$ (see Table II). In both cases, for $\mathbf{C I}_{\text {plan }}$ we obtain negative second derivatives (for the remaining modes we obtain only positive second derivatives) for the curvature along the curvilinear coordinate $t_{i}$. Thus, $\mathbf{C I}_{\text {plan }}$ is the analog of a second-order saddle point in the intersection space (i.e., on a hyperline). Displacement along a combination of the bond inversion (gradient difference) and methylene torsion coordinate lowers the energies of the two states but preserves the degeneracy. The same applies for the combination of pyramidalization and gradient difference coordinates.

Along the curved methylene torsion plus gradient difference coordinate, we have optimized a lower-lying intersec-

TABLE IV. Relevant intersection space frequencies (RT-II profiles, Fig. 1) at $\mathrm{CI}_{\text {plan }}$ and $\mathrm{CI}_{\text {perp }}$.

\begin{tabular}{lcc}
\hline \hline $\mathrm{CI}_{\text {plan }}$ & $\begin{array}{c}{ }^{1} A_{1} \text { state } \\
\text { Symmetry }\end{array}$ & $\begin{array}{c}{ }^{1} B_{2} \text { state } \\
\omega\left(\mathrm{cm}^{-1}\right)\end{array}$ \\
\hline$b_{1}{ }^{\mathrm{a}}$ & 405 & $320 i$ \\
$a_{2}{ }^{\mathrm{b}}$ & 431 & $181 i$ \\
$\mathrm{CI}_{\text {perp }}$ & ${ }^{1} A_{2}$ state & ${ }^{1} B_{1}$ state \\
Symmetry & $\omega\left(\mathrm{cm}^{-1}\right)$ & $\omega\left(\mathrm{cm}^{-1}\right)$ \\
\hline$a_{2}{ }^{\mathrm{b}}$ & $1006 i$ & 376 \\
$b_{1}{ }^{\mathrm{a}}$ & $20 i$ & 176 \\
\hline
\end{tabular}

${ }^{a}$ Methylene pyramidalization.

${ }^{\mathrm{b}}$ Methylene torsion.

$\mathrm{c}_{i}=\sqrt{-1}$. 


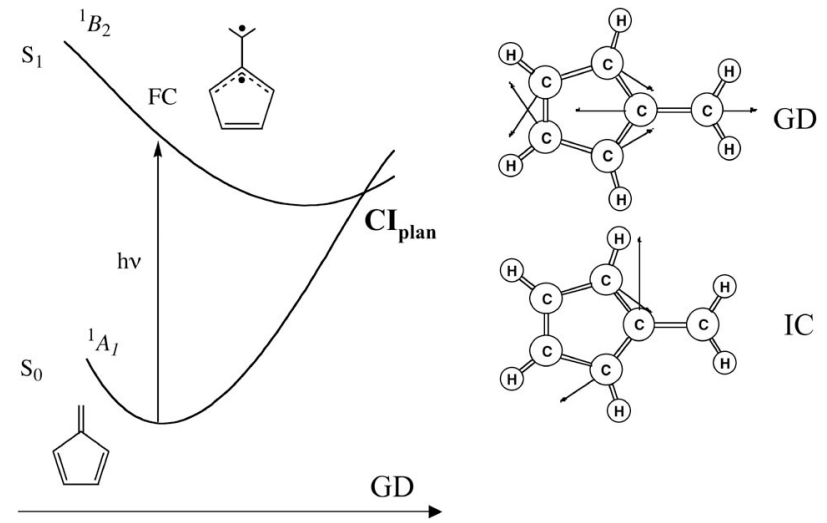

FIG. 4. Surface topology for the $S_{0}$ and $S_{1}$ states in the restricted space of $C_{2 v}$ symmetry (planar geometries), including branching-space coordinates at $\mathbf{C I}_{\text {plan }}$.

tion of $C_{2}$ symmetry ( $A$ and $B$ states) with a methylene torsion angle of $63^{\circ}, \mathbf{C I}_{63}$, which lies approximately $10 \mathrm{kcal} \mathrm{mol}^{-1}$ below the $\mathbf{C I}_{\text {plan }}$. This confirms the prediction of our intersection space Hessian analysis. Further, along the curved pyramidalization mode, there is a conical intersection of $C_{s}$ symmetry, $\mathbf{C I}_{\text {pyr }}$, which lies approximately $1.5 \mathrm{kcal} \mathrm{mol}^{-1}$ below the planar one. Analysis of the Hessians at $\mathbf{C I}_{63}$ and $\mathbf{C I}_{\text {pyr }}$ gives positive second derivatives along all curved coordinates $t_{i}$, see Table II.

To complete our analysis of the CI hyperline, we have calculated the Hessians at the twisted intersection of $C_{2 v}$ symmetry, $\mathbf{C I}_{\text {perp }}$. In this case, the two degenerate states have $A_{2}$ and $B_{1}$ symmetries, and correlate with the $A_{1}$ and $B_{2}$ states at the planar intersection, respectively. Similar to the results for the planar intersection, at the twisted intersection of $C_{2 v}$ symmetry, there is substantial second-order splitting (RT-II-type profile) along the methylene torsion and pyramidalization modes (of $a_{2}$ and $b_{1}$ symmetries respectively) (see Table IV). However, the curvature of the $A$ and $B$ states along the rectilinear intersection space coordinates is reversed compared to $\mathbf{C I}_{\text {plan }}$. The large imaginary frequency for the $A_{2}$ state along the torsion mode comes from the $\mathrm{C}_{1}-\mathrm{C}_{6} \pi$ bond for that state, and the gradient difference coordinate corresponds, as in the case of the planar intersection, to the symmetric bond inversion. Using Eq. (18), we find a negative sign for $\left(\partial^{2} E_{s}\left(t_{i}\right) / \partial t_{i}^{2}\right)_{t_{i}=0}$ along the curved coordinate of combined bond inversion and torsion, and a positive sign along the one that contains the methylene pyramidalization (Table II). Thus, $\mathbf{C I}_{\text {perp }}$ is a first-order saddle point on the CI hyperline. This critical point connects the twisted intersection $\mathbf{C I}_{63}$ with its analog $\mathbf{C I}_{63}^{\prime}$ (torsion angle $117^{\circ}$ ) and lies approximately $2 \mathrm{kcal} \mathrm{mol}^{-1}$ above them (see the energetics of Table I).

To summarize, our CI hyperline analysis for the two conical intersections of $C_{2 v}$ symmetry gives RT-II-type profiles along the methylene torsion and pyramidalization modes for both structures. These are the only two modes that give a large second-order splitting at these points. While there is no intuitive way of guessing whether a lowering of symmetry along these modes will lead to lower-energy coni-

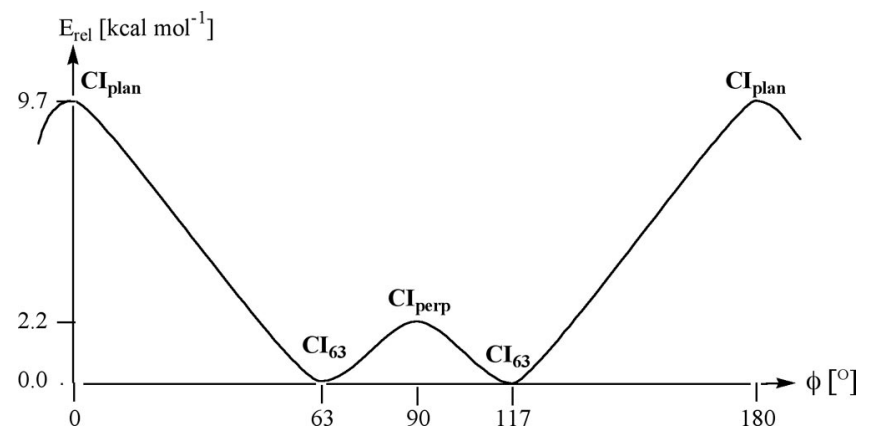

FIG. 5. One-dimensional profile (projection) of the seam of the $S_{1} / S_{0}$ intersection in fulvene along the curved methylene torsion/bond-inversion coordinate.

cal intersection structures, with the help of Eq. (18) it is possible to predict this behavior.

We now discuss how the computed curvature of the hyperline-together with the first- and second-order parameters collected in Table II-can be used to produce a global "cartoon" of the two potential surfaces (Figs. 3 and 5) showing the seam of intersection. Figure 5 shows a onedimensional representation of the seam along the curvilinear coordinate composed of the methylene torsion/bond inversion. The curvilinear coordinate is projected onto the torsion angle $\phi$ and the profile corresponds to half a rotation of the methylene group $\left(180^{\circ}\right)$. The $C_{2 v}$ structures $\left(\phi=0^{\circ}, 90^{\circ}\right.$, and $180^{\circ}$ ) are maxima along this curvilinear coordinate, whereas the $C_{2}$ structures $\left(\phi=63^{\circ}\right.$ and $117^{\circ}$ ) are minima. Figure 3 is a two-dimensional cartoon of the $S_{0}$ and $S_{1}$ surfaces in the space of one rectilinear intersection space coordinate, the torsion, and the bond-inversion coordinate (gradient difference). This cartoon illustrates the curvatures of the two states at the two critical points of $C_{2 v}$ symmetry. From Fig. 3, it is clear that the seam of intersection lies along a curved line, a combination of the bond stretching and methylene torsion coordinates. Along the path from $\mathbf{C I}_{\text {plan }}$ to $\mathbf{C I}_{\text {perp }}$ (through $\mathbf{C I}_{63}$ ), the bond lengths change progressively, following the bond-inversion coordinate. This is shown by the bond lengths in Table III (stretching of the $\mathrm{C}_{1}-\mathrm{C}_{2}$, $\mathrm{C}_{1}-\mathrm{C}_{5}$, and $\mathrm{C}_{3}-\mathrm{C}_{4}$ bonds and contraction of $\mathrm{C}_{1}-\mathrm{C}_{6}$, $\mathrm{C}_{2}-\mathrm{C}_{3}$, and $\mathrm{C}_{4}-\mathrm{C}_{5}$ ). At the same time, the gradient difference coordinate changes along the seam. It is purely bond inversion at the $C_{2 v}$ structures (where the gradient along the methylene torsion is zero), but it has a torsion component all along the seam (cf. the gradient difference at the $C_{2}$ minimum, Fig. 6). Thus, the two rectilinear coordinates mix along the CI hyperline.

In a similar manner, the path connecting the $\mathbf{C I}_{\text {plan }}$ and $\mathbf{C I}_{\text {pyr }}$ also contains the bond-inversion coordinate, but gradually gains a pyramidalization component along the curvilinear seam. See Fig. 7 for the gradient difference coordinate at the optimized intersection $\mathbf{C I}_{\text {pyr }}$.

To summarize, the branching and intersection space coordinates provide a rectilinear set of orthogonal coordinates that can be used to characterize the curvilinear conical intersection seam. The rectilinear coordinates are tangent to the curved seam at any optimized critical point on the CI seam. Using Eq. (18) above allows the determination of the curva- 


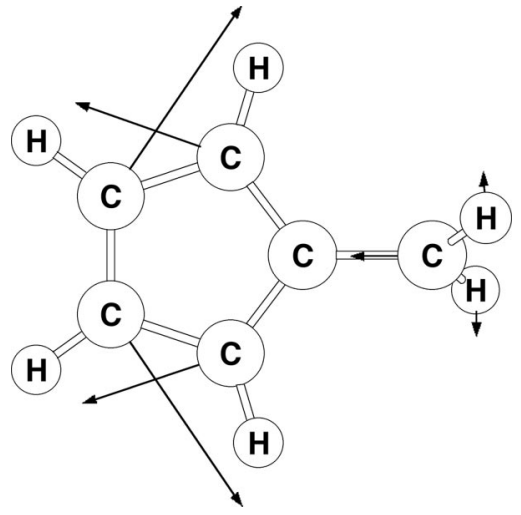

FIG. 6. Gradient difference vector at $\mathbf{C I}_{63}$.

ture of the seam at these points and predicts whether or not there are any lower-lying CI critical points. In fulvene, we have shown that the two previously found points of $C_{2 v}$ symmetry are in fact first- and second-order saddle points on the CI hyperline and accordingly there exist two minima on the hyperline that had not been found previously.

\section{CONCLUSIONS}

Our CI hyperline analysis of fulvene has shown that at the optimized conical intersections of $C_{2 v}$ symmetry, there are substantial second-order splittings associated with Renner-Teller type-II profiles along some vibrational coordinates. These second-order splittings along the rectilinear intersection space coordinates can be readily rationalized in terms of a valence-bond representation of the degenerate states. The rectilinear branching and intersection space coordinates are tangent to the seam of intersection at any optimized CI critical point; however, they can be used to determine the local curvature of the seam. In fulvene, this analysis was used to show that the $C_{2 v}$ CI critical points found in a previous study are in fact first- and second-order saddle points on the hyperline. Our new methodology predicts the curvilinear seam coordinate that retains the degeneracy and leads to the minima on the hyperline, which we have optimized for the first time.

Our analysis presented above remains valid as long as the cross-quadratic terms ${ }^{c} \delta \gamma_{i j}$ and $\eta_{i j}^{A B}(i \neq j)$ are zero. In

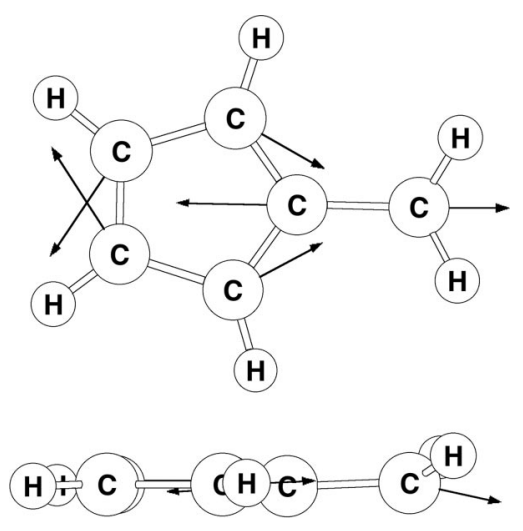

FIG. 7. Gradient difference vector at $\mathbf{C I}_{\mathrm{pyr}}$. that case, the coordinates that compose the $(3 N-8)$-dimensional degenerate space only have to be redefined as the curvilinear coordinates $t_{i}$. This is not valid anymore when there are large cross-quadratic terms, i.e., displacements along two curved coordinates $t_{i}$ and $t_{j}$ will not retain degeneracy. In our analysis, we cannot identify the cross-quadratic terms directly, but their effect can be seen on the eigenvectors of the intersection space Hessian (the rectilinear "normal modes" of the intersection space), that will be significantly different for the Hessians of the two states. In these cases, one should consider that the degenerate space at the conical intersection has a lower dimension than $(3 \mathrm{~N}$ $-8)$. Future work will consider the application of the above methodology to cases with no symmetry when this effect may occur.

\section{ACKNOWLEDGMENTS}

All the computations were carried out on an IBM-SP2 funded jointly by IBM (U.K.) and HEFCE (U.K.). One of the authors (L.B.) is financed by the Ramón y Cajal program from the Spanish Ministerio de Ciencia y Tecnología (MCyT) and by Grant Nos. BQU2002-04112-C02-02 and BQU2002-03334 from the Dirección General de Investigación (MCyT).

\section{APPENDIX: MODIFIED HYPERLINE CURVATURE}

In this Appendix we give a calculation of the second derivative of the hyperline with the inclusion of ${ }^{a} \delta \gamma_{1}$ terms (second-order splitting along the gradient difference). Equation (13), which is used to determine the curved coordinate $t_{i}$ becomes

$$
\delta \kappa \bar{Q}_{x_{1}}+{ }^{a} \delta \gamma_{1} \bar{Q}_{x_{1}}^{2}+{ }^{c} \delta \gamma_{i} \bar{Q}_{i}^{2}=0
$$

Two cases have to be considered here. For the first case ( $\delta \gamma_{1} \delta \gamma_{i}>0$, elliptical seam), the second derivative is

$$
\left(\frac{\partial^{2} E\left(t_{i}\right)}{\partial t_{i}^{2}}\right)_{t_{i}=0}=\frac{\delta \kappa}{2^{a} \delta \gamma_{1}{ }^{c} \delta \gamma_{i}}\left(\gamma_{i i}^{A} \kappa^{B}-\gamma_{i i}^{B} \kappa^{A}\right) .
$$

For the alternative case ( $\delta \gamma_{1} \delta \gamma_{i}<0$, hyperbolic seam), the second derivative is

$$
\left(\frac{\partial^{2} E\left(t_{i}\right)}{\partial t_{i}^{2}}\right)_{t_{i}=0}=\frac{\delta \kappa}{2^{a} \delta \gamma_{1}{ }^{c} \delta \gamma_{i}}\left(\gamma_{i i}^{B} \kappa^{A}-\gamma_{i i}^{A} \kappa^{B}\right) .
$$

In both cases, the sign of the second derivative is given by

$$
\operatorname{sign}\left(\frac{\partial^{2} E\left(t_{i}\right)}{\partial t_{i}^{2}}\right)_{t_{i}=0} \equiv \operatorname{sign}\left[\delta \kappa\left(\gamma_{i i}^{A} \kappa^{B}-\gamma_{i i}^{B} \kappa^{A}\right)\right]
$$

${ }^{1}$ G. J. Atchity, S. S. Xantheas, and K. J. Ruedenberg, J. Chem. Phys. 95, 1862 (1991)

${ }^{2}$ M. Klessinger and J. Michl, Excited States and Photochemistry of Organic Molecules (VCH, New York, 1995).

${ }^{3}$ D. R. Yarkony, Rev. Mod. Phys. 68, 985 (1996).

${ }^{4}$ D. R. Yarkony, Acc. Chem. Res. 31, 511 (1998).

${ }^{5}$ D. R. Yarkony, J. Phys. Chem. A 105, 6277 (2001).

${ }^{6}$ F. Bernardi, M. Olivucci, and M. A. Robb, Chem. Soc. Rev. 25, 321 (1996).

${ }^{7}$ M. A. Robb, M. Garavelli, M. Olivucci, and F. Bernardi, A Computational 
Strategy for Organic Photochemistry, in Reviews in Computational Chemistry, edited by K. B. Lipkowitz and D. B. Boyd (Wiley-VCH, New York, 2000), Vol. 15, pp. 87-146.

${ }^{8}$ M. Olivucci and A. Migani, in Conical Intersections: Electronic Structure, Dynamics and Spectroscopy, edited by W. Domcke, D. Yarkony, and H. Köppel (World Scientific, Singapore, 2004), Vol. 15.

${ }^{9}$ A. Sanchez-Galvez, P. A. Hunt, M. A. Robb, M. Olivucci, T. Vreven, and H. B. Schlegel, J. Am. Chem. Soc. 122, 2911 (2000).

${ }^{10}$ M. Ben-Nun, J. Quenneville, and T. J. Martinez, J. Phys. Chem. A 104, $5161(2000)$.

${ }^{11}$ M. Boggio-Pasqua, M. Ravaglia, M. J. Bearpark, M. Garavelli, and M. A. Robb, J. Phys. Chem. A 107, 11139 (2003).

${ }^{12}$ C. Ko, B. Levine, A. Toniolo, L. Manohar, S. Olsen, H.-J. Werner, and T. J. Martinez, J. Am. Chem. Soc. 125, 12710 (2003).

${ }^{13}$ O. Weingart, A. Migani, M. Olivucci, M. A. Robb, V. Buss, and P. Hunt, J. Phys. Chem. A 108, 4685 (2004)

${ }^{14}$ A. Migani, M. A. Robb, and M. Olivucci, J. Am. Chem. Soc. 125, 2804 (2003).

${ }^{15}$ M. J. Bearpark, F. Bernardi, M. Olivucci, M. A. Robb, and B. R. Smith, J. Am. Chem. Soc. 118, 5254 (1996).

${ }^{16}$ J. Von-Neumann and E. Wigner, Z. Phys. 30, 467 (1929).

${ }^{17}$ H. Köppel, W. Domcke, and L. S. Cederbaum, Adv. Chem. Phys. 57, 59 (1984).

${ }^{18}$ T. A. Barckholtz and T. A. Miller, Int. Rev. Phys. Chem. 17, 435 (1998).
${ }^{19}$ T. A. Barckholtz and T. A. Miller, J. Phys. Chem. A 103, 2321 (1999).

${ }^{20}$ I. N. Ragazos, M. A. Robb, F. Bernardi, and M. Olivucci, Chem. Phys. Lett. 197, 217 (1992).

${ }^{21}$ M. J. Bearpark, M. A. Robb, and H. B. Schlegel, Chem. Phys. Lett. 223, 269 (1994).

${ }^{22}$ T. J. Lee, D. J. Fox, H. F. Schaeffer III, and R. M. Pitzer, J. Chem. Phys. 81, 356 (1984).

${ }^{23}$ D. R. Yarkony, J. Chem. Phys. 108, 3200 (2004).

${ }^{24}$ M. J. Frisch, G. W. Trucks, H. B. Schlegel et al., Gaussian 99 (Revison C1) Pittsburgh, 1999.

${ }^{25}$ S. F. Boys, Rev. Mod. Phys. 32, 296 (1960).

${ }^{26}$ L. Blancafort, P. Celani, M. J. Bearpark, and M. A. Robb, Theor. Chem. Acc. 110, 92 (2003).

${ }^{27}$ M. Beer and H. C. Longuet-Higgins, J. Chem. Phys. 23, 1390 (1955).

${ }^{28}$ See EPAPS Document No. E-JCPSA6-121-019445 for Cartesian coordinates of all optimized fulvene CI points, together with matched harmonic vibrational frequencies for the intersection space and a valence-bond analysis of each component of the degenerate electronic state based upon the spin-exchange density. A direct link to this document may be found in the online article's HTML reference section. The document may also be reached via the EPAPS homepage (http://www.aip.org/pubservs/ epaps.html) or from ftp.aip.org in the directory /epaps/. See the EPAPS homepage for more information. 